\title{
The relation of the pleural thickening in tuberculosis pleurisy with the activity of adenosine deaminase
}

\author{
B. Uskul1, H. Turker1, C. Ulman1, M. Ertugrul1, A. Selvi1, \\ A. Kant ${ }^{1}$, S. Arslan1, M. Ozgel2
}

ABSTRACT: The relation of the pleural thickening in tuberculosis pleurisy with the activity of adenosine deaminase. B. Uskul, H. Turker, C. Ulman, M. Ertugrul, A. Selvi, A. Kant, S. Arslan, M. Ozgel.

Background: Residual pleural thickening (RPT) still occurs in most patients with tuberculosis pleurisy despite advances in the treatment of tuberculosis. The aim of this study was to evaluate the significance of RPT in tuberculosis pleurisy with the patients clinical findings, biochemical and microbiological properties of pleural effusion and with the total adenosine deaminase (ADA) and isoenzymes levels.

Methods: 121 tuberculosis pleurisy patients were evaluated retrospectively. According to posteroanterior chest $\mathrm{x}$-rays, the $63(52 \%)$ cases with the thickness $2 \mathrm{~mm}$ or more in lower lateral hemithorax were grouped as I and the $\mathbf{5 8}$ (48\%) cases without pleural thickness were grouped as II. The amount of pleural effusion was classified into small, medium or massive according to their chest $x$-rays. In both groups; sex, age, symptoms score, bacteriological and biochemical tests and ADA levels were recorded.

Results: $81(67 \%)$ male and $40(33 \%)$ female, overall 121 patients were enrolled into the study. RPT was found higher in males $(p=0.014)$ and the increase ran parallel with the amount of cigarette smoking $(p=0.014)$. RPT was found to be lower in small effusions $(p=0.001)$. The group with RPT, the serum albumin was found lower $(p=0.002)$, pleural fluid total protein $(p=0.047)$ and the ratio of pleural fluid protein to serum protein $(\mathrm{p}=\mathbf{0 . 0 0 2})$ were found higher. In group I, total ADA: 69.5 $\pm 38.9 \mathrm{IU} / \mathrm{L}$ and ADA2: 41.3 $\pm 31.6 \mathrm{IU} / \mathrm{L}$ were higher than the cases without RPT $(\mathrm{p}=0.032, \mathrm{p}=0.017$, respectively).

Conclusions: We suggest that the immunological mechanisms are effective in the development of pleural thickening.

Monaldi Arch Chest Dis 2005; 63: 2, 101-107.

Keywords: Tuberculosis pleurisy, pleural thickening, adenosine deaminase.

${ }^{1}$ Department of Pulmonology, SSK Sureyyapasa Center for Chest Diseases and Thoracic Surgery, Istanbul,

2 Department of Biochemistry, SSK Sureyyapasa Center for Chest Diseases and Thoracic Surgery, Istanbul, Turkey

Correspondence: Bahadir Uskul, MD, Zumrutevler mah. Nil cad. Hukukcular sitesi A-3 blok Da:8 Maltepe Istanbul 84352 Turkey; e-mail: tbuskul@yahoo.com

\section{Introduction}

Pleural involvement is one of the most frequent extrapulmonary manifestations of tuberculosis [1]. Residual pleural thickening still occurs in most patients with tuberculosis despite advances in the treatment of tuberculosis [2-5]. To date, the relationship between pleural thickening and biochemical, biological and microbiological parameters of the pleural fluid have been studied. No significant correlation, however, has been found between these parameters (for example protein level, lactate dehydrogenase and glucose level and $\mathrm{pH}$ of the pleural fluid) and pleural thickening so far [2, 4]. Furthermore, it is well known that RPT is not related to chemotherapeutic regimens, therapeutic thoracentesis and adding corticosteroids to the treatment $[2,3,6,7]$.

We aimed in the present study to investigate the clinical findings and biochemical and microbiological characteristics of pleural fluid as well as the relationship between residual pleural thickening and levels of total adenosine deaminase and several isoenzymes of ADA in the patients with tuberculous pleurisy (TP).

\section{Methods}

A total of 158 patients were diagnosed as with TP between January 2002 and December 2002 in our centre. The diagnosis of TP was based on the following criteria: (a) Granulomatous inflammation with necrosis on the pleural biopsy samples. (b) Not considering an alternative diagnosis in the presence of granulomatous inflammation without necrosis on the pleural biopsy samples; (c) Growth of M.tuberculosis in the pleural fluid and/or pleural biopsy cultures. 24 of 158 patients were excluded from the study as they had received corticosteroids in addition to the anti-tuberculous therapy. A further 6 patients were excluded from the study because of the lack of follow-up information and an additional 7 patients because of inadequate medical records. Medical records of the remaining 121 patients were reviewed retrospectively. The following information was obtained from the med- 
ical records: the age of the patient, tobacco consumption (package/year), duration of the symptoms (day), history of past tuberculosis, index patient, the presence or absence of pleuritic chest pain, cough, fever, fatigue, weight loss, sputum, night sweating and dyspnea, reaction to intradermal purified protein derivative, hemoglobin level, leukocyte count, erythrocyte sedimentation rate, serum levels of total protein, albumin, glucose and lactate dehydrogenase, pleural fluid levels of protein, albumin, glucose and LDH and activities of total ADA and isoenzymes 1 and 2 of ADA, cellular composition of the pleural fluid and the size of the effusion. The size of effusion was classified as small, medium or massive according to the area occupied by the effusion on a posteroanterior chest radiograph. Additionally, whether parenchymal infiltration existed on the PA chest radiograph was also recorded. Additionally, histological characteristics and culture results of the pleural biopsy samples and culture results of the pleural fluid samples and direct acid fast bacillus investigations and culture results of the sputum were also recorded.

All patients were treated with appropriate anti-tuberculous regimens for sufficient duration $(6$ to 12 months). The anti-tuberculosis regimens given were as follows: 112 (93\%) patients received isoniazid and rifampin for 6 months with ethambutol and pyrazinamide for the first 2 months; $6(5 \%)$ patients received isoniazid, rifampin and ethambutol for 9 months with streptomycin for the first 2 months due to hepatotoxicity; and $3(2 \%)$ patients received isoniazid and rifampin for 12 months with ethambutol and pyrazinamide for the first 2 months. 121 patients were separated into two groups based on their chest roentgenograms at the end of treatment. $63 \mathrm{pa}-$ tients in group I had residual pleural thickening that was defined as a pleural thickening greater than $2 \mathrm{~mm}$. RPT was measured in the lower lateral hemithorax of a posteroanterior chest radiograph at the level of an imaginary line intersecting the diaphragmatic dome. 58 patients in group II had no such thickening.

\section{Statistical Analysis}

Statistical analyses were performed using SPSS software for windows (version 11.0). The data is expressed as the mean \pm the standard deviation unless otherwise noted. Parameter distributions were compared with a normal distribution using the Kolmogorov-Smirnov test. For abnormal distributions, the groups were compared by the Mann-Whitney $U$ test, and for normal distributions, by Student's $t$ test. Qualitative variables were compared with the chi-square $\left(\chi^{2}\right)$ method and Yates' correction. The Fisher Exact Test was used as needed.

\section{Results}

$81(67 \%)$ of 121 patients with TP were male (mean age: $36 \pm 16.2)$ and $40(33 \%)$ were female (mean age: $33 \pm 11.8$ ). Mean age of all patients was $35 \pm 14.9$. The rate of the patients who had RPT following the anti-tuberculous therapy was $52 \%$. Comparisons between the clinical characteristics of $63 \mathrm{pa}-$ tients with RPT and 58 patients without RPT were given in table 1 . Pleural thickening was significantly higher in male patients $(p=0.014)$. Mean age of the patients with RPT was $37 \pm 16.4$ and that of the patients without RPT in the group II was $34 \pm 13.1$. There was no statistically significant difference between two groups in terms of age, history of previous tuberculosis infections, index cases, duration of the symptoms and PPD values. There was no significant difference between the two groups in terms of clini-

Table 1. - Comparison of Clinical Characteristics in Patients with Residual Pleural Thickening (Group I) and Those without Pleural Thickening (Group II) *

\begin{tabular}{|c|c|c|c|}
\hline & $\begin{array}{c}\text { Group I } \\
\mathrm{n}(\%)\end{array}$ & $\begin{array}{c}\text { Group II } \\
\mathrm{n}(\%)\end{array}$ & $\mathrm{p}$ \\
\hline Age, year $\S$ & $37 \pm 16.4$ & $34 \pm 13.1$ & NS \\
\hline Male & $49 / 63(78)$ & $32 / 58(55)$ & 0.014 \\
\hline History of past tuberculosis & $4 / 63(6)$ & $2 / 58(3)$ & NS \\
\hline Patient index & $15 / 63(24)$ & $19 / 58(33)$ & NS \\
\hline Duration of the symptoms (days) $\dagger$ & $15[2-150]$ & $17.5[7-120]$ & NS \\
\hline Pleuritic chest pain & $34 / 63(54)$ & $49 / 58(84)$ & NS \\
\hline Cough & $44 / 63(70)$ & $40 / 58(69)$ & NS \\
\hline Fever & $30 / 63(48)$ & $24 / 58(41)$ & NS \\
\hline Fatigue & $19 / 63(30)$ & $14 / 58(24)$ & NS \\
\hline Weight loss & $28 / 63(44)$ & $18 / 58(31)$ & NS \\
\hline Sputum & $14 / 63(22)$ & $14 / 58(24)$ & NS \\
\hline Night sweats & $22 / 63(35)$ & $26 / 58(45)$ & NS \\
\hline Dyspnea & $33 / 63(52)$ & $19 / 58(33)$ & NS \\
\hline $\mathrm{PPD} \geq 15 \mathrm{~mm} \neq$ & $13 / 47(28)$ & $12 / 51(23)$ & NS \\
\hline
\end{tabular}

* Values given as No. of patients / total No. of patients (\%), unless otherwise indicated.

$\S$ Values given as mean \pm SD.

$\dagger$ Values given as median [min-max].

$\$$ Data unavailable for 16 patients in group I and 7 patients in group II.

NS not significant. 
cal findings. But dyspnea was more frequent in the group I and pleuritic chest pain was more frequent in the group II. With regard to the smoking rates of the groups, the proportions of the patients who were not used to smoke were almost equal in the two groups (60\% in the group I and $59 \%$ in the group II). RPT increased with rate of smoking $(p=0.014)$ (table 2$)$.

Diagnosis was made by means of a pleural biopsy in $94 \%$ of the patients, whereas 2 patients were reported to have chronic pleuritis. Pleural biopsy could not be completed in two patients because of a defense developed during procedure. These two patients had their diagnoses with growth of M.tuberculosis in pleural fluid cultures. M.tuberculosis was isolated from the pleural fluid cultures in 2 of the 42 patients $(5 \%)$ in group I and 5 of $(11 \%) 47$ patients in group II. M.tuberculosis was isolated from pleural biopsy samples in 2 of 8 patients $(25 \%)$ in the group I whereas no pleural biopsy sample of any patient in the group II was submitted to microbiology laboratory for culture. No statistically significant difference was found between the results of pleural biopsy and culture when these results were compared (table 3 ).

Three of the patients who developed pleural thickening $(5 \%)$ underwent decortication. Two of these three patients were male and one of them was female. Pleural thickening was on the right side in two patients and on the left side in one patient. Two of these three patients had moderate restriction and one had severe restriction on respiratory function tests. Postoperative diagnoses of these three patients were reported as granulomatous inflammation with necrosis.

Lymphocyte rate was investigated in the cytological examinations of pleural fluid samples in 88 of 121 patients $(73 \%)$. Lymphocyte rate in the pleural fluid was above $82 \%$ in 56 (64\%) of these patients. With regard to distribution of these patients in the groups, the lymphocyte rate was above $82 \%$ in 39 of 44 patients $(89 \%)$ in the group of pleural thickening and in only 17 of 44 patients $(39 \%)$ in the group without RPT ( $p=0.0001)$ (table 4).

Table 5 shows the comparisons in radiological and microbiological findings between the two groups. Effusion sizes on PA chest roentgenograms were classified as small, medium and massive. The rate of pleural thickening was less in small effusions $(\mathrm{p}=0.001)$. Only two patients had bilateral effusion. Although residual thickening was more frequent in effusions on the left side, the difference was not statistically significant. Effusions were ac-

Table 2. - Smoking Rates of the Groups

\begin{tabular}{lccc}
\hline & $\begin{array}{c}\text { Group I } \\
\mathrm{n}(\%)\end{array}$ & $\begin{array}{c}\text { Group II } \\
\mathrm{n}(\%)\end{array}$ & $\mathrm{p}$ \\
\hline Non-smoker & $38(60)$ & $34(59)$ & $\mathrm{NS}$ \\
Smoker & $25(40)$ & $24(41)$ & $\mathrm{NS}$ \\
Tobacco consumption, pk/yr & $22 \pm 19.1$ & $11 \pm 9.9$ & 0.014 \\
\hline
\end{tabular}

NS: not significant.

Table 3. - Comparison of Histological and Microbiologic Characteristics in Patients with Residual Pleural Thickening (Group I) and those without Pleural Thickening (Group II)

\begin{tabular}{lccc}
\hline & $\begin{array}{c}\text { Group I } \\
\mathrm{n}(\%)\end{array}$ & $\begin{array}{c}\text { Group II } \\
\mathrm{n}(\%)\end{array}$ & $\mathrm{p}$ \\
\hline Pleural biopsy & & & $\mathrm{NS}$ \\
$\quad$ Caseating granulomata & $49 / 63(78)$ & $47 / 56(84)$ & $\mathrm{NS}$ \\
$\quad$ Non-caseating granulomata & $14 / 63(22)$ & $7 / 56(12)$ & $\mathrm{NS}$ \\
$\quad$ Chronic pleuritis & $0(0)$ & $2 / 56(4)$ & $\mathrm{NS}$ \\
Diagnosis with first biopsy & $59 / 63(94)$ & $53 / 56(95)$ & $5 / 47(11)$ \\
Positive pleural fluid culture & $2 / 42(5)$ & $0(0)$ & \\
Positive pleural biopsy culture & $2 / 8(25)$ & $0(0)$ & \\
Decortication & $3 / 63(5)$ & & \\
\hline
\end{tabular}

NS: not significant.

Table 4. - Lymphocyte Rates in the Cytological Examinations of Pleural Fluid Samples

\begin{tabular}{lccc}
\hline $\begin{array}{l}\text { Total lymphocye } \\
\text { rates in pleural fluid }\end{array}$ & $\begin{array}{c}\text { Group I } \\
\mathrm{n}(\%)\end{array}$ & $\begin{array}{c}\text { Group II } \\
\mathrm{n}(\%)\end{array}$ & $\begin{array}{c}\text { Total } \\
\mathrm{n}(\%)\end{array}$ \\
\hline$<82 \%$ & $5(11)$ & $27(61)$ & $32(36)$ \\
$\geq \% 82$ & $39(89)$ & $17(39)^{*}$ & $56(64)$ \\
Total (No.) & 44 & 44 & 88 \\
\hline$* \mathrm{p}=0.0001$. & & &
\end{tabular}


companied by parenchymal infiltrations on PA chest roentgenograms in 27 patients (23\%). These were in the form of sequel fibrotic lesions on bilateral apexes in 3 patients, non-cavitary infiltration in 15 patients (unilateral in 8 patients and bilateral in 6 , miliar in another) and cavitary infiltration in 6 patients (unilateral in 3 patients, bilateral in other 3 patients). In 49 patients, acido-resistant bacteria was investigated in the sputum and microbial culture was carried out in these patients. AFB results were positive in two patients while 16 patients had positive culture result. Two patients with positive AFB result also had cavitary infiltration with effusion.

Table 6 shows the biochemical and biological parameters in the blood and pleural fluid samples of the patients in the group I and II. Statistical comparisons of these parameters were given in table 7. Serum albumin level was found to be lower and total protein in the pleural fluid to be higher in the group with RPT ( $=0.002$ and $\mathrm{p}=0.047$, respectively). The rate of protein level in the pleural fluid to serum protein level (pTpr/sTpr rate) was also higher in the group with pleural thickening $(p=0.002)$. Total level of ADA and level of ADA2 isoenzyme were statistically significantly higher in the group with pleural thickening $(\mathrm{p}=0.032$ and $p=0.017$, respectively). There was no significant difference between groups in ADA-1 isoenzyme levels. No statistically significant differences were found between groups in other biochemical parameters in both pleural fluid and blood.

Table 5. - Comparison of Roentgenographic Findings in Patients with Residual Pleural Thickening (Group I) and those without Pleural Thickening (Group II)

\begin{tabular}{lccc}
\hline & $\begin{array}{c}\text { Group I } \\
\mathrm{n}(\%)\end{array}$ & $\begin{array}{c}\text { Group II } \\
\mathrm{n}(\%)\end{array}$ & $\mathrm{p}$ \\
\hline Initial size of pleural effusion & & & \\
$\quad$ Small & $22 / 63(35)$ & $41 / 58(71)$ & 0,001 \\
$\quad$ Medium & $37 / 63(59)$ & $14 / 58(24)$ & \\
$\quad$ Massive & $4 / 63(6)$ & $3 / 58(5)$ & $\mathrm{NS}$ \\
Localisation of pleural effusion & $22 / 63(35)$ & $38 / 58(65)$ & \\
$\quad$ Right & $41 / 63(65)$ & $18 / 58(31)$ & $\mathrm{NS}$ \\
$\quad$ Left & $0(0)$ & $2 / 58(3)$ & $\mathrm{NS}$ \\
$\quad$ Bilateral & $2 / 63(3)$ & $1 / 58(2)$ & $\mathrm{NS}$ \\
Accompanied by parenchymal infiltrations & $8 / 63(13)$ & $7 / 58(12)$ & $\mathrm{NS}$ \\
$\quad$ Sequel fibrotic lesions & $2 / 63(3)$ & $4 / 58(7)$ & $\mathrm{NS}$ \\
$\quad$ Non-cavitary infiltration & $1 / 26(4)$ & $1 / 23(4)$ & $9 / 23(39)$ \\
Cavitary infiltration & $7 / 26(27)$ & & \\
$\quad$ AFB results positive & & \\
Positive culture result & & \\
\hline
\end{tabular}

$\mathrm{AFB}=$ Acid fast bacillus.

NS: not significant.

Table 6. - Comparison of Serum and Fluid Characteristics in Patients with Residual Pleural Thickening (Group I) and Those without Pleural Thickening (Group II) ‡

\begin{tabular}{lcc}
\hline & Group I & Group II \\
\hline Haemoglobin, g/dl & $12.4 \pm 1.6$ & $12.4 \pm 1.6$ \\
WBC 103 & $7.2 \pm 2.3$ & $7.1 \pm 1.6$ \\
ESR mm/h & $76.3 \pm 28.7$ & $80.3 \pm 26.1$ \\
Serum LDH†, IU/L & $252[70-632]$ & $239[121-779]$ \\
Serum Glucose $\dagger$, mg/dL & $96[60-440]$ & $95[65-167]$ \\
Serum Total protein, g/dL & $7.2 \pm 0.6$ & $7.3 \pm 0.6$ \\
Serum Albumin, g/dL & $4.0 \pm 0.6$ & $4.4 \pm 0.5$ \\
Pleural LDH†, IU/L & $575[156-2280]$ & $608[61-3690]$ \\
Pleural Glucose $\dagger, \mathrm{mg} / \mathrm{dL}$ & $86.5[10-430]$ & $88[26-161]$ \\
Pleural Total protein, g/dL & $5.5 \pm 0.8$ & $5.2 \pm 0.7$ \\
Pleural Albumin, g/dL & $3.1 \pm 0.5$ & $3.2 \pm 0.5$ \\
Total ADA, IU/L & $69.5 \pm 38.9$ & $55.8 \pm 25.4$ \\
ADA1, IU/L & $29.4 \pm 14.4$ & $27.3 \pm 10.9$ \\
ADA2, IU/L & $41.3 \pm 31.6$ & $28.7 \pm 20.8$ \\
\hline
\end{tabular}

$\ddagger$ Values given as mean $\pm \mathrm{SD}$, unless otherwise indicated.

$\dagger$ Values given as median [min-max]. 
Table 7. - Statistical Comparisons of Biochemical and Biological Parameters *

\begin{tabular}{lccc}
\hline & Group I & Group II & $\mathrm{p}$ \\
\hline Serum Albumin, g/dL & $4.0 \pm 0.6$ & $4.4 \pm 0.5$ & 0.002 \\
Pleural Total protein, g/dL & $5.5 \pm 0.8$ & $5.2 \pm 0.7$ & 0.047 \\
Pleural Total protein / Serum Total protein $\dagger$ & 67.97 & 48.36 & $0.002 \ddagger$ \\
Total ADA, IU/L & $69.5 \pm 38.9$ & $55.8 \pm 25.4$ & 0.032 \\
ADA1, IU/L & $29.4 \pm 14.4$ & $27.3 \pm 10.9$ & $\mathrm{NS}$ \\
ADA2, IU/L & $41.3 \pm 31.6$ & $28.7 \pm 20.8$ & 0.017 \\
\hline
\end{tabular}

* Values given as mean $\pm \mathrm{SD}$, unless otherwise indicated.

$\dagger$ Values given as mean rank.

$\ddagger$ The groups were compared by the Mann-Whitney $U$ test.

NS: not significant.

\section{Discussion}

Tuberculous pleurisy (TP) ranks second only to lymphatic involvement in terms of extrapulmonary involvement in tuberculosis with a rate of $23 \%$ to $40 \%$. At the beginning of the century, TP was considered as delayed type of hypersensitivity reaction rather than pleural infection [8]. Mycobacterium tuberculosis invades the pleural cavity mainly through rupture of subpleural caseificating foci within 6 to 12 weeks following the primary infection. Patients with TP invariably have a small subpleural nidus of tuberculosis showing fibrous and granulomatous inflammation and clear signs of leakage into the pleural space [9].

Bacillus protein antigens seem to induce a delayed hypersensitivity reaction that stimulates lymphocytes, which in turn release certain lymphokines that (1) activate macrophages against the mycobacterium and (2) alter the permeability of pleural vessels and affect the formation of granulomas [10]. The fact that pleural fluid and biopsy cultures are usually negative in the patients with TP is another finding supporting the delayed type hypersensitivity reaction [11]. TP is more common in children and young adults in communities in which it is endemic whereas it is seen in elder individuals (elder than 30) in the communities in which the incidence of tuberculosis is lower [12]. A study on TP found the mean age of the patients with TP to be 47 years. This was explained with more common occurrence of post-primary infection, increased likelihood of extrapulmonary tuberculous infections with "Human Immune Deficiency Virus" and increased incidence of primary infection in elder populations [1]. The mean age of the patients in our series was 35 and $41 \%$ of the patients were younger than 30 while $50 \%$ were elder than 30 and $9 \%$ were elder than 60 . This may be interpreted as reduced incidence of tuberculosis in our country.

TP is usually unilateral and the volume of fluid accumulated in TP is often in small to moderate size [11]. Only 2 of our patients (2\%) had bilateral effusion. Pleural thickening was less common in the patients with less fluid in our series and this difference was statistically significant $(\mathrm{p}=0.001)$. As the volume of pleural fluid increases the pleur- al thickening increases and dyspnea becomes more prominent among the clinical symptoms. However, there was no statistically significant difference to demonstrate this.

In our series, $97 \%$ of the patients had their diagnoses with closed pleural biopsy and M.tuberculosis could be isolated at a very low rate in pleural fluid and biopsy cultures. This is a finding in favour of delayed type hypersensitivity reaction in the pathogenesis of TP.

Pleural thickening continues in some patients following completion of the treatment. The rate of development of sequel has been reported to be between $10 \%$ and $50 \%$ in literature [2-5]. Pleural thickening should be evaluated for decortication if it is causing severe alteration in respiratory functions and the fluid persists despite aggressive treatments, or empyema has developed [13]. The rate of development of pleural thickening was found to be $52 \%$ in our series and only 3 patients (2\%) required decortication. These figures are consistent with the literature.

The relationship examined has been between the bacteriological and biochemical parameters of pleural fluid and RPT in the studies performed so far. Although the level of LDH in the pleural fluid is a reliable indication of pleural inflammation, no significant relationship has thus far been found between pleural thickening and LDH level [14]. If pleural thickening is not related to the biochemical findings of the pleural fluid or therapeutic regimens, what could it be related to? This question was asked previously by Barbas et al and they considered that delayed type hypersensitivity reaction rather than inflammatory response against the infection was implicated for pleural fibrosis [2]. A significant relationship was found between the presence of pleural thickening and levels of tumour necrosis factor and lysosyme in a study conducted in 1997. The authors of this study argued that high TNF concentration increased the deposition of collagen in the pleura and this might cause pleural fibrosis by increasing fibroblast proliferation and stimulation [4].

Adenosine deaminase is a polymorphic enzyme involved in the purine metabolism. It catalyses the reaction that deaminates adenosine to inosine and deoxyinosine [15]. Adenosine deaminase 
comprises 2 isoenzymes: ADA1, which is found all cells, and ADA2, which is found only in monocytes and macrophages [16].

ADA activity is highest in the lymphoid tissue. Its activity is 10 to 20 times higher in T lymphocytes than in the B lymphocytes $[15,17]$. ADA has been used in the diagnosis of tuberculous pleural effusions for years. ADA2 has a higher positive predictive value (92\%) and higher sensitivity (93\%) and specificity $(92 \%)$ for tuberculous effusion than ADA and ADA1 [18]. In pleural fluids it reflects the cellular immune response in the pleural compartment, especially the activation of $\mathrm{T}$ lymphocytes [19].

A recent study reported that increased activity of the enzyme ADA is mainly due to the increased level of ADA2 and it has been suggested that ADA2 activity was an indication of turn-over or activity of monocytes and macrophages [20, 21]. A recent study suggested that the inflammatory changes caused by M.tuberculosis in the pleural cavity effectively stimulate the production of ADA2 as ADA2 levels are much higher in the pleural effusion than in the serum of patients with TP [22]. This high ADA2 activity is caused by increased production by monocytes and macrophages that have been stimulated by live phagocytosed microorganisms [23]. Lymphocytes are the predominant cells in the cytological examination of the pleural fluid. The presence of lymphocytes in the pleural fluid at a rate of more than $90 \%$ is a finding supporting the diagnosis of TP [24]. Total lymphocyte rate was statistically significant in our series, especially in the group with RPT. In tuberculous pleural effusions both the absolute number and percentage of T-lymphocytes are significantly higher than in the peripheral blood, a fact which could explain the increase in adenosine deaminase activity, an enzyme predominantly detected in T-cells [25].

Higher levels of total ADA and ADA-2 seen in the group with pleural thickening in the present study ( $\mathrm{p}=0.032$ and $\mathrm{p}=0.017$, respectively) have suggested that immunological mechanism might also play a role in the development of pleural thickening. Especially statistically significant difference between two groups in ADA2 levels suggesting that activated $\mathrm{T}$ lymphocytes play an effective role in the development of pleural thickening. Higher lymphocyte rates in the group with pleural thickening also support our opinion. Although statistically not significant, the fact that the proportion of the patients with a PPD reaction $\geq 15$ $\mathrm{mm}$ was more in the group with pleural thickening also suggests that delayed type hypersensitivity, that is T lymphocytes plays an efficient role.

The fact that protein levels of the patients with pleural thickening were higher in the pleural fluid than in the serum might be explained by the impairment of permeability of pleural membrane that was more severe in these patients. Changes in the permeability of the pleural vessels are due to the lymphokines released from the activated lymphocytes [10].

Although the present study is a retrospective one, the relationship we found between total ADA and ADA2 activities and pleural thickening suggests that the immunological mechanisms are effective in the development of pleural thickening.

\section{References}

1. Seibert AF, Haynes J Jr, Middleton R, Bass JB Jr. Tuberculous pleural effusion. Twenty years experince. Chest 1991; 99: 883-886.

2. Barbas CSV, Cuiker A, de Varvalho CRR, et al. The relationship between pleural fluid findings and the development of pleural thickening in patients with pleural tuberculosis. Chest 1991; 100: 1264-1267.

3. Wyser C, Walzl G, Smedema JP, et al. Corticosteroids in the treatment of tuberculous pleurisy: a double-blind, placebo-controlled, randomized study. Chest 1996; 110: 333-338.

4. De Pablo A, Villena V, Echave-Sustaeta J, et al. Are pleural fluid parameters related to the development of residual pleural thickening in tuberculosis? Chest 1997; 112: 1293-1297.

5. Candela A, Andujar J, Hernandez L, et al. Functional sequelae of tuberculous pleurisy in patients correctly treated. Chest 2003; 123: 1996-2000.

6. Galarza I, Canete C, Granados A, et al. Randomised trial of corticosteroids in the treatment of tuberculous pleurisy. Thorax 1995; 50: 1305-1307.

7. Lee $\mathrm{CH}$, Wang WJ, Lan RS, et al. Corticosteroids in the treatment of tuberculous pleurisy. A double-blind, placebo-controlled, randomized study. Chest 1988; 94 : 1256-1259.

8. Paterson HC. The pleural reaction to inoculation with tubercle bacilli in vaccinated and normal guinea pigs. Am Rev Tuberculous Pulmon Dis 1917; 1: 353

9. Stead WW, Eichenhola A, Stauss HK. Operative and pathologic findings in twenty-four patients with syndrome of idiopathic pleurisy with effusion, presumably tuberculous. Am Rev Respir Dis 1955; 71: 473-502.

10. Yamamoto S, Dunn CJ, Willoughby DA. Studies on delayed hypersensitivity pleural exudates in guinea pigs. II. The interrelationship of monocytic and lymphocytic cells with respect to migration activity. Immunology 1976; 30: 513-519.

11. Berger HW, Mejia E. Tuberculous pleurisy. Chest 1973; 63: 88-92.

12. Chan CHS, Arnold M, Chan CY, et al. Clinical and pathological features of tuberculous pleural effusions and its long-term consequences. Respiration 1991; 58: 171-175.

13. Light RW. Tuberculous pleural effusions. In: Light RW (ed). Pleural disease. 4th ed. Lippincott Williams and Wilkins, 2001: 182-195.

14. Light RW. Diagnostic principles in pleural disease. Eur Respir J 1997; 10: 476-481

15. Van der Weyden MB, Kelley WN. Human adenosine deaminase distribution and properties. $\mathrm{J}$ Biol Chem 1976; 251: 5448-5456.

16. Gakis C. Adenosine deaminase (ADA) isoenzymes ADA1 and ADA2: diagnostic biological role. Eur Respir J 1996; 9: 632-633.

17. Barton RW, Goldshneider I. Nucleotide metabolizing enzymes and lymphocytic differentiation. Mol Cell Biochem 1979; 28: 135-147.

18. Kamholz SL. Pleural tuberculosis. In: Rom WN, Garay SM (eds). Tuberculosis. Philadelphia: Williams and Wilkins, 2004: 497-507.

19. Ocana I, Martinez-Vazquez JM, Segura RM, et al. Adenosine deaminase in pleural fluids. Test for diagnosis of tuberculous pleural effusion. Chest 1983; 84: 51-53.

20. Ungerer JPJ, Oosthuizen HM, Retief JH, et al. Significance of adenosine deaminase activity and its isoen- 
zymes in tuberculous effusions. Chest 1994; 106: 33-37

21. Valdes L, Alvarez D, San Jose E, et al. Tuberculous pleurisy A study of 254 patients. Arch Intern Med 1998; 158: $2017-2021$

22. Shibagoki T, Hasegama Y, Saito H, et al. Adenosine deaminase isozymes in tuberculous pleural effusion. $J$ Lab Clin Med 1996; 127: 348-352.
23. Ungerer JPJ, Oosthuizen HM, Bissbort SH, et al. Serum adenosine deaminase: isoenzymes and diagnostic application. Clin Chem 1992; 38: 1322-1326.

24. Sahn SA. The pleura (state of art). Am Rev Respir Dis 1988; 138: 184-234.

25. Petterson T, Klockars M, Hellstrom PE, et al. T and B lymphocytes in pleural effusions. Chest 1978; 73: 49.

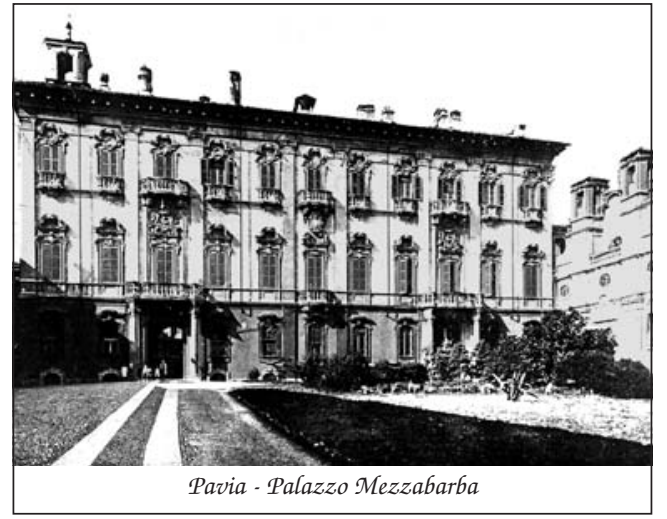

